\title{
Bayesian Updating of Parameters for a Sediment Entrainment Model via Markov Chain Monte Carlo
}

\author{
Fu-Chun $\mathrm{Wu}^{1}$ and C. C. Chen ${ }^{2}$
}

\begin{abstract}
A Bayesian framework incorporating Markov chain Monte Carlo (MCMC) for updating the parameters of a sediment entrainment model is presented. Three subjects were pursued in this study. First, sensitivity analyses were performed via univariate MCMC. The results reveal that the posteriors resulting from two- and three-chain MCMC were not significantly different; two-chain MCMC converged faster than three chains. The proposal scale factor significantly affects the rate of convergence, but not the posteriors. The sampler outputs resulting from informed priors converged faster than those resulting from uninformed priors. The correlation coefficient of the Gram-Charlier (GC) probability density function (PDF) is a physical constraint imposed on MCMC in which a higher correlation would slow the rate of convergence. The results also indicate that the parameter uncertainty is reduced with increasing number of input data. Second, multivariate MCMC were carried out to simultaneously update the velocity coefficient $C$ and the statistical moments of the GC PDF. For fully rough flows, the distribution of $C$ was significantly modified via multivariate MCMC. However, for transitional regimes the posterior values of $C$ resulting from univariate and multivariate MCMC were not significantly different. For both rough and transitional regimes, the differences between the prior and posterior distributions of the statistical moments were limited. Third, the practical effect of updated parameters on the prediction of entrainment probabilities was demonstrated. With all the parameters updated, the sediment entrainment model was able to compute more accurately and realistically the entrainment probabilities. The present work offers an alternative approach to estimating the hydraulic parameters not easily observed.
\end{abstract}

DOI: 10.1061/(ASCE)0733-9429(2009)135:1(22)

CE Database subject headings: Bayesian analysis; Parameters; Markov chains; Monte Carlo method; Sediment; Entrainment.

\section{Introduction}

The Markov chain Monte Carlo (MCMC) method generates random samples that can be used to evaluate marginal and conditional probabilities. The underlying principle of MCMC is simple: to sample randomly from a "target" probability distribution, then design a Markov chain whose long-time equilibrium (or stationary state) follows that distribution, run it for a time long enough to be confident that an approximate equilibrium has been attained, then record the state of the Markov chain as an approximate draw from the equilibrium. The MCMC method was first introduced by statistical physicists (Metropolis et al. 1953) using a symmetric Markov chain. Over the last two decades MCMC has become increasingly popular, primarily attributed to the contribution of Gelfand and Smith (1990) by showing the effective applications of MCMC in Bayesian problems. A vast literature demonstrates the power of MCMC in dealing with problems ranging from image processing to geophysics to bioinformatics. Among the excellent reviews of MCMC are the article by Spall (2003) and the

${ }^{1}$ Professor, Dept. of Bioenvironmental Systems Engineering, Hydrotech Research Inst., and Center for Ecological Engineering, National Taiwan Univ., Taipei 106, Taiwan, ROC. E-mail: fcwu@ntu.edu.tw

${ }^{2}$ Graduate Research Assistant, Dept. of Bioenvironmental Systems Engineering, National Taiwan Univ., Taipei 106, Taiwan, ROC.

Note. Discussion open until June 1, 2009. Separate discussions must be submitted for individual papers. The manuscript for this paper was submitted for review and possible publication on March 3, 2007; approved on April 15, 2008. This paper is part of the Journal of Hydraulic Engineering, Vol. 135, No. 1, January 1, 2009. CASCE, ISSN 07339429/2009/1-22-37/\$25.00. books devoted to MCMC (Gilks et al. 1996; Gelman et al. 2004; Gamerman and Lopes 2006).

In hydrology, MCMC is also frequently employed to deal with the Bayesian problems (e.g., Balakrishnan et al. 2003; Marshall et al. 2004; Reis and Stedinger 2005; Renard et al. 2006). To date, applications of MCMC in the hydraulic engineering are surprisingly sparse. This probably stems from the fact that Bayesian inference exhibits little resemblance to the deterministic approaches conventionally adopted by hydraulic engineers for the task of parameter estimation. Moreover, the MCMC method and its applications in hydraulics have been rarely demonstrated.

This work presents a Bayesian framework in which we employ MCMC to update the parameters of a sediment entrainment model by incorporating the data of entrainment probabilities. The posterior distributions of the parameters are applied in prediction of sediment entrainment, and the practical improvements resulting from this study are demonstrated. The work presented here resembles the "inverse modeling approach" used in the area of subsurface hydrology for refining uncertain parameters with additional input information, and offers an alternative approach to estimating the hydraulic parameters that are not easily observed.

\section{Overview of Sediment Entrainment Model}

A sediment entrainment model that incorporated the near-bed coherent flow structures was proposed by Wu and Yang (2004a) and later applied to investigate the role of turbulent bursting in entrainment of mixed-size sediment (Wu and Jiang 2007). Here, only the key parts of this model are summarized, and readers are referred to the original work for details. The model consists of 
two major components, i.e., the probabilistic and mechanistic submodels. The former mainly deals with the near-bed coherent flow structures that were characterized by a third-order GramCharlier (GC) joint probability density function (PDF) of streamwise and vertical turbulent fluctuations. Random samples are drawn from the GC joint PDF to construct the pairs of instantaneous velocities approaching a sediment particle. The velocity pairs are then used in the mechanistic submodel to evaluate the instantaneous hydrodynamic forces acting on a particle of the ith size fraction randomly configured on the mixed-size sediment bed and the corresponding probabilities of entrainment. The procedure is implemented over the full range of each random variable to estimate the expected value of entrainment probability for the ith size fraction, denoted as $P T_{i}$. The input variables include bed shear stress $\tau_{0}$, grain size $D_{i}$ and proportion $p_{i}$ of the ith fraction $(i=1, \ldots, n ; n=$ total number of size fractions).

In the probabilistic submodel, the third-order GC joint PDF of two-dimensional turbulent fluctuations is given by

$$
g(U, V)=\phi\left(U, V \mid \mathbf{0}, \mathbf{\Sigma}_{U V}\right) \cdot\left(1+L_{1}+L_{2}+L_{3}+L_{4}\right)
$$

in which $U=u^{\prime} / \sigma_{u}$ and $V=v^{\prime} / \sigma_{v}=$ normalized velocity fluctuations in the streamwise and vertical directions, respectively, where $\sigma_{u}$ and $\sigma_{v}=$ standard deviations of $u^{\prime}$ and $v^{\prime}$, respectively; $\phi\left(U, V \mid \mathbf{0}, \boldsymbol{\Sigma}_{U V}\right)=$ bivariate standard normal (SN) joint PDF with zero mean vector $\mathbf{0}=[0,0]^{T}$ and covariance matrix $\boldsymbol{\Sigma}_{U V}$, which is expressed by

$$
\Sigma_{U V}=\left[\begin{array}{cc}
1 & R_{u v} \\
R_{u v} & 1
\end{array}\right]
$$

where $R_{u v}=\overline{u^{\prime} v^{\prime}} / \sigma_{u} \sigma_{v}=$ correlation coefficient. The near-bed values of $R_{u v}$ typically range between -0.4 and -0.5 (Pope 2000; $\mathrm{Wu}$ and Yang 2004a). A value of $R_{u v}=-0.45$ was used herein; two alternative values, -0.4 and -0.5 , were also used for sensitivity analyses. The SN joint PDF of $U$ and $V$ can be written as

$$
\phi\left(U, V \mid \mathbf{0}, \boldsymbol{\Sigma}_{U V}\right)=\frac{1}{2 \pi \sqrt{1-R_{u v}^{2}}} \exp \left[-\frac{U^{2}-2 R_{u v} U V+V^{2}}{2\left(1-R_{u v}^{2}\right)}\right]
$$

The third-order expansion of Hermite polynomials, $\left(1+L_{1}+L_{2}\right.$ $\left.+L_{3}+L_{4}\right)$, is defined by

$$
\begin{gathered}
L_{1}=\frac{S_{u}}{3 !}\left[-\left(\frac{R_{v}}{\left(1-R_{u v}^{2}\right)^{2}}\right)^{3}+\frac{3 R_{v}}{\left(1-R_{u v}^{2}\right)^{2}}\right] \\
L_{2}=\frac{M_{21}}{2 !}\left[\frac{3 R_{u v} U-2 R_{u v}^{2} V-V}{\left(1-R_{u v}^{2}\right)^{2}}-\frac{R_{u} R_{v}^{2}}{\left(1-R_{u v}^{2}\right)^{3}}\right] \\
L_{3}=\frac{M_{12}}{2 !}\left[\frac{3 R_{u v} V-2 R_{u v}^{2} U-U}{\left(1-R_{u v}^{2}\right)^{2}}-\frac{R_{u}^{2} R_{v}}{\left(1-R_{u v}^{2}\right)^{3}}\right] \\
L_{4}=\frac{S_{v}}{3 !}\left[-\left(\frac{R_{u}}{\left(1-R_{u v}^{2}\right)^{2}}\right)^{3}+\frac{3 R_{u}}{\left(1-R_{u v}^{2}\right)^{2}}\right]
\end{gathered}
$$

where $R_{u}=R_{u v} U-V ; R_{v}=R_{u v} V-U ; S_{u}=\overline{u^{\prime 3}} / \sigma_{u}^{3} ;$ and $S_{v}=\overline{v^{\prime 3}} / \sigma_{v}^{3}$ =skewness factors; and $M_{21}=\overline{u^{\prime 2} v^{\prime}} / \sigma_{u}^{2} \sigma_{v}$ and $M_{12}=\overline{u^{\prime} v^{\prime 2}} / \sigma_{u} \sigma_{v}^{2}$ $=$ diffusion factors. In this submodel, the parameter values to be specified include the second- and third-order statistical moments, i.e., $\sigma_{u}, \sigma_{v}, S_{u}, S_{v}, M_{21}$, and $M_{12}$, which are briefly described in the following.

Based on an analysis of the compiled data, $\mathrm{Wu}$ and Yang (2004a) recommended that for transitional flows $\left(k_{s}^{+}<70\right)$, $\sigma_{u} / u_{*}=-0.187 \ln \left(k_{s}^{+}\right)+2.93$ and $S_{u}=0.102 \ln \left(k_{s}^{+}\right)$, where $k_{s}^{+}$ $=$ roughness Reynolds number $=u_{*} k_{s} / \nu ; u_{*}=\sqrt{\tau_{0} / \rho}=$ bed shear velocity; $\rho=$ density of fluid; $k_{s}=2 D_{50} ; D_{50}=$ median size; and $\nu=$ kinematic viscosity. For fully rough flows $\left(k_{s}^{+} \geqslant 70\right)$, constant values of $\sigma_{u} / u_{*}=2.1$ and $S_{u}=0.43$ were observed. Wu and Jiang (2007) reanalyzed the compiled data set and further suggested that $\sigma_{v} / u_{*}=0.99, S_{v}=-0.01, M_{21}=-0.07$, and $M_{12}=0.12$ for both transitional and fully rough flows. The limited data available for these analyses give rise to uncertainties in the parameter values, and we seek to update these parameters using a Bayesian MCMC approach.

In the mechanistic submodel, characterization of the near-bed velocity profile is crucial for evaluations of hydrodynamic forces and the resulting probabilities of entrainment. The structure of the near-bed region can be characterized by a roughness layer in the close proximity of the bed surface and a logarithmic layer above the roughness layer (Nikora et al. 2001). In the roughness layer, the double-averaged (i.e., time and spatially averaged) streamwise velocity is described by a linear profile, i.e.

$$
\frac{\bar{u}(y)}{u_{*}}=C\left(\frac{y}{\delta}\right) \text { for } y \leqslant \delta
$$

where $\bar{u}(y)=$ time-averaged streamwise velocity at a height $y$ from the origin, which is located at a distance $0.25 D_{84}$ below the mean bed surface; $\delta=$ thickness of the roughness layer, $\delta$ is taken to be the sand diameter for a uniform sand bed, whereas it is $1.5 D_{50}$ for a mixed-size gravel bed. Nikora et al. (2001) suggested that for a gravel bed the velocity coefficient $C$ is in the range between 5.3 and 5.6, whereas for a sand bed $C \approx 8.5$. The velocity profile in the logarithmic layer follows the universal log distribution, which can be expressed as

$$
\frac{\bar{u}(y)}{u_{*}}=C+\frac{1}{\kappa} \ln \left(\frac{y}{\delta}\right) \quad \text { for } y>\delta
$$

where $\kappa=$ von Karman constant $=0.4$. As shown in Eqs. (5) and (6), the coefficient $C$ affects the mean velocity profiles in both the roughness and logarithmic layers, to which a protruding particle is exposed. As an instantaneous velocity can be decomposed as a sum of mean velocity and fluctuation, the instantaneous drag, lift, and turning moment exerted on a particle would vary sensitively with the value of $C$ used, which in turn affects the probabilities of entrainment. By incorporating the compiled data, we seek to update model parameters via a Bayesian framework in which MCMC is used as a sampler to derive posterior distributions.

\section{Markov Chain Monte Carlo}

In the context of Bayesian inference, the posterior distribution of model parameters that incorporates both the prior knowledge and additional information is given by

$$
P(\boldsymbol{\theta} \mid \mathbf{d})=\frac{P(\boldsymbol{\theta}) P(\mathbf{d} \mid \boldsymbol{\theta})}{\int P(\boldsymbol{\theta}) P(\mathbf{d} \mid \boldsymbol{\theta}) d \boldsymbol{\theta}} \propto P(\boldsymbol{\theta}) P(\mathbf{d} \mid \boldsymbol{\theta})
$$

in which $P(\boldsymbol{\theta} \mid \mathbf{d})=$ posterior distribution of model parameters $\boldsymbol{\theta}$ given additional data $\mathbf{d} ; P(\boldsymbol{\theta})=$ prior distribution of $\boldsymbol{\theta} ; P(\mathbf{d} \mid \boldsymbol{\theta})$ $=$ likelihood of $\mathbf{d}$ under condition $\boldsymbol{\theta}$, where $\boldsymbol{\theta}=$ parameter vector; $\mathbf{d}=m \times n$ matrix of observed data, $m=$ number of observations, and $n=$ number of data in each observation. It has been demonstrated (Gilks et al. 1996; Gelman et al. 2004; Gamerman and 
Lopes 2006) that the normalizing constant in the denominator of Eq. (7) can be discarded such that a tractable relation of proportionality (without having to integrate over the parameter domains) would be obtained. In general, evaluation of the posterior distribution $P(\boldsymbol{\theta} \mid \mathbf{d})$ is not straightforward because it is usually not possible to sample from the likelihood. However, it is possible to calculate the likelihood for a given "realization of model parameters," and MCMC exploits this property to generate samples from the posterior distribution when the chain has converged. A sufficiently large number of these samples can be then used as a good numerical approximation to the target posterior distribution $P(\boldsymbol{\theta} \mid \mathbf{d})$.

\section{Metropolis Algorithm}

A Metropolis algorithm using a symmetric proposal distribution was employed in this study to construct the Markov chain because of its simplicity and efficiency. The Metropolis algorithm samples a candidate parameter vector $\boldsymbol{\theta}_{*}$ from a symmetric proposal distribution $q\left(\boldsymbol{\theta}_{*} \mid \boldsymbol{\theta}_{t}\right)$ and then obtains the $(t+1)$ th realization of parameter vector, $\boldsymbol{\theta}_{t+1}$, with an acceptance-rejection procedure. The acceptance-rejection procedure is based on comparisons of a random sample $\zeta$ drawn from $\operatorname{Uniform}(0,1)$ with an acceptance probability $\alpha$ evaluated by

$$
\alpha=\frac{P\left(\boldsymbol{\theta}_{*} \mid \mathbf{d}\right) q\left(\boldsymbol{\theta}_{t} \mid \boldsymbol{\theta}_{*}\right)}{P\left(\boldsymbol{\theta}_{t} \mid \mathbf{d}\right) q\left(\boldsymbol{\theta}_{*} \mid \boldsymbol{\theta}_{t}\right)}=\frac{P\left(\boldsymbol{\theta}_{*}\right) P\left(\mathbf{d} \mid \boldsymbol{\theta}_{*}\right)}{P\left(\boldsymbol{\theta}_{t}\right) P\left(\mathbf{d} \mid \boldsymbol{\theta}_{t}\right)}
$$

where the proposals are canceled out as $q\left(\boldsymbol{\theta}_{*} \mid \boldsymbol{\theta}_{t}\right)=q\left(\boldsymbol{\theta}_{t} \mid \boldsymbol{\theta}_{*}\right)$ for symmetric distributions. The Markov chain so generated would eventually converge to the target posterior given any form of proposals, provided that the Markov chain is "ergodic," which means that the states of the chain have sufficiently experienced the whole parameter space (Gilks et al. 1996). To implement the Metropolis algorithm, the prior $P\left(\boldsymbol{\theta}_{t}\right)$, likelihood $P\left(\mathbf{d} \mid \boldsymbol{\theta}_{t}\right)$, proposal $q\left(\boldsymbol{\theta}_{*} \mid \boldsymbol{\theta}_{t}\right)$, along with the convergence diagnostics must be specified, as described in subsequent sections.

\section{Prior Distributions}

For the velocity coefficient $C$, an uninformed (i.e., unbounded uniform) prior was used. We also used two alternative priors for sensitivity analyses, which included a normal distribution $N(8.05,3.1)$ with mean and variance determined from the lower and upper bounds reported by Nikora et al. (2001), and a posterior normal PDF $N(17.3,5.5)$ resulting from univariate MCMC and uninformed prior. For the six statistical moments of the GC PDF, MCMC simulations were carried out on all parameters in fully rough flows, but only on four parameters in transitional flows where $\sigma_{u} / u_{*}$ and $S_{u}$ vary deterministically as a function of $k_{s}^{+}$. For fully rough flows, a multivariate normal PDF $N\left(\boldsymbol{\theta}_{6} \mid \boldsymbol{\mu}_{\text {prior }}, \boldsymbol{\Sigma}_{\text {prior }}\right)$ was used as the prior of the parameters $\boldsymbol{\theta}_{6}=\left[\sigma_{u} / u_{*}, \sigma_{v} / u_{*}\right.$, $\left.S_{u}, S_{v}, M_{21}, M_{12}\right]^{T}$, where $\boldsymbol{\mu}_{\text {prior }}$ and $\boldsymbol{\Sigma}_{\text {prior }}=$ prior mean vector and covariance matrix derived from the compiled data set ( $\mathrm{Wu}$ and Yang 2004a; Wu and Jiang 2007), and are given by

$$
\boldsymbol{\mu}_{\text {prior }}=\left[\begin{array}{llllll}
2.1, & 0.99, & 0.43, & -0.01, & -0.07, & 0.12
\end{array}\right]^{T}
$$

$$
\Sigma_{\text {prior }}=\left[\begin{array}{cccccc}
0.0021 & & & & & \\
& 0.056 & & & & \\
& & 0.018 & & & \\
& & & 0.054 & & \\
& & & & 0.01 & \\
& & & & & 0.0026
\end{array}\right]
$$

Because the compiled data revealed no significant correlations among these parameters (Wu and Jiang 2007), they were assumed independent for simplicity, thus Eq. (9b) was literally a variance matrix. For transitional flow regimes, the parameter vector and normal prior were replaced by $\boldsymbol{\theta}_{4}=\left[\sigma_{v} / u_{*}, S_{v}, M_{21}, M_{12}\right]^{T}$ and $N\left(\boldsymbol{\theta}_{4} \mid \boldsymbol{\mu}_{\text {prior }}, \boldsymbol{\Sigma}_{\text {prior }}\right)$, in which $\boldsymbol{\mu}_{\text {prior }}$ and $\boldsymbol{\Sigma}_{\text {prior }}$ were modified from Eq. (9) accordingly.

\section{Likelihood Function}

The likelihood $P(\mathbf{d} \mid \boldsymbol{\theta})$ is a conditional probability of observing $\mathbf{d}$ given parameters $\boldsymbol{\theta}$. Here, we followed Box (1980) and Rubin (1984) by assuming that the observed entrainment probabilities of the $i$ th fraction, $E T_{i}$, are normally distributed with a mean $P T_{i}$ and a variance $\sigma^{2}$, where $P T_{i}=$ predicted entrainment probability, $\sigma^{2}=$ quantifier of model errors, evaluated with the discrepancies between observed and predicted results. Following Wu and Jiang (2007) gave an estimate of $\sigma^{2}=0.001$. Assuming independency among these distributions leads to

$$
P(\mathbf{d} \mid \boldsymbol{\theta})=\prod_{k=1}^{m} \prod_{i=1}^{n} N\left[E T_{i}^{k} \mid P T_{i}^{k}(\boldsymbol{\theta}), \sigma^{2}\right]
$$

where $k=$ observation index and $n=$ number of size fractions. It should be noted that the assumption of independent normal distributions is reasonable because each of these distributions describes a relation between observed and predicted results but not a relation between observations or size fractions. Eq. (10) was used by Eq. (8) to account for the differences between predicted and observed entrainment probabilities.

\section{Proposal Distributions}

As mentioned earlier, any proposal distribution (also called candidate-generating, probing, or jumping distribution) will ultimately deliver samples from the target posterior. The rate of convergence, however, depends on the resemblance between proposal and target distributions. In this study we employed a random-walk Metropolis algorithm and Gaussian proposals that have good convergence properties (Draper 2006). The proposal distributions of $C$ can be expressed as $q\left(\boldsymbol{\theta}_{*} \mid \boldsymbol{\theta}_{t}\right)=N\left(C_{*} \mid C_{t}, s^{2}\right)$, where $s=$ scale factor. The scale factor must be specified carefully. A cautious proposal (with a small $s$ ) that generates small steps $\left(\boldsymbol{\theta}_{*}-\boldsymbol{\theta}_{t}\right)$ will generally have high acceptance rate $\alpha$, but the chain will converge slowly. A bold proposal (with a large $s$ ) generating large steps will often propose moves from the body to the tails of the distribution, giving small values of $\alpha$, that in turn will prevent a chain from moving and again result in slow convergence (Gilks et al. 1996). For the velocity coefficient $C$, a value of $s=0.5$ was used. Two alternative values of $s=0.25$ and 1.0 were also used for sensiti- 
vity analyses. For the six statistical moments of the GC PDF, the variance matrix of the proposal was taken to be $0.2 \boldsymbol{\Sigma}_{\text {prior }}$, and the proposal distribution was given by $q\left(\boldsymbol{\theta}_{*} \mid \boldsymbol{\theta}_{t}\right)=N\left(\boldsymbol{\theta}_{6, *} \mid \boldsymbol{\theta}_{6, t}\right.$, $\left.0.2 \Sigma_{\text {prior }}\right)$. A relation $q\left(\boldsymbol{\theta}_{*} \mid \boldsymbol{\theta}_{t}\right)=q\left(\boldsymbol{\theta}_{*}-\boldsymbol{\theta}_{t}\right)$ holds for symmetric proposals, thus the proposals of $C$ and $\boldsymbol{\theta}_{6}$ can be rewritten as

$$
\begin{gathered}
N\left(C_{*} \mid C_{t}, s^{2}\right)=N\left(C_{*}-C_{t} \mid 0, s^{2}\right) \\
N\left(\boldsymbol{\theta}_{6, *} \mid \boldsymbol{\theta}_{6, t}, 0.2 \Sigma_{\text {prior }}\right)=N\left(\boldsymbol{\theta}_{6, *}-\boldsymbol{\theta}_{6, t} \mid \mathbf{0}, 0.2 \Sigma_{\text {prior }}\right)
\end{gathered}
$$

Random samples were drawn from these proposals and used as candidates to advance the chains subject to the acceptancerejection procedure.

\section{Convergence Diagnostics}

The purpose of convergence diagnostics is to determine when it is reasonable to believe that the samples generated by MCMC simulations are representative of the underlying equilibrium distribution. To assess whether a chain has converged to the stationary distribution, we employed a widely used diagnostic metric proposed by Brooks and Gelman (1998), who suggested using multiple chains obtained with overdispersed starting values. Throughout this study, we used two chains to carry out MCMC simulations; however, a three-chain MCMC was also performed for sensitivity analyses. The diagnostic metric is based on the calculation of potential scale reduction factor (PSRF) $R$, which is defined as the ratio between total variance and within-sequence variance. If convergence is reached, the between-sequence variance should become negligible, leading to a value of $R \approx 1$. Usually, values of $R \leqslant 1.1$ are considered as acceptable (Gelman et al. 2004). However, for multivariate chains with dimensionality $>5$, a convergence criterion $R \leqslant 1.5$ has been recommended (Brooks and Gelman 1998). In this study, the first diagnostic was performed at 500 iterations; thereinafter convergence was diagnosed every 50 iterations. The convergence was confirmed by checking if the chains that met the criterion have lasted for at least 500 iterations. The posterior distribution was then obtained by discarding the initial burn-in iterations and using the converged portions. In addition, the autocorrelation function was examined to see if the autocorrelations within the sampler output was negligible (Smith 2005).

\section{Procedure}

The flowchart of the multiple-chain MCMC is shown in Fig. 1, where the input data and specifications of prior, likelihood, and proposal were given at the beginning. The first chain was started at a specified value, which was used by the sediment entrainment model to compute the probabilities of entrainment (for all observed events and size fractions). A candidate parameter was drawn from the proposal and again used to compute the probabilities of entrainment. These predicted values and observed data were used to calculate the acceptance probability $\alpha$ via Eq. (8), and $\alpha$ was used in the acceptance-rejection procedure moving the chain to the next state. These steps were repeated first for all chains and then for iterations. When all chains had advanced to a point where the convergence was confirmed as described earlier, the output posterior distribution was obtained using the converged portions of all chains.

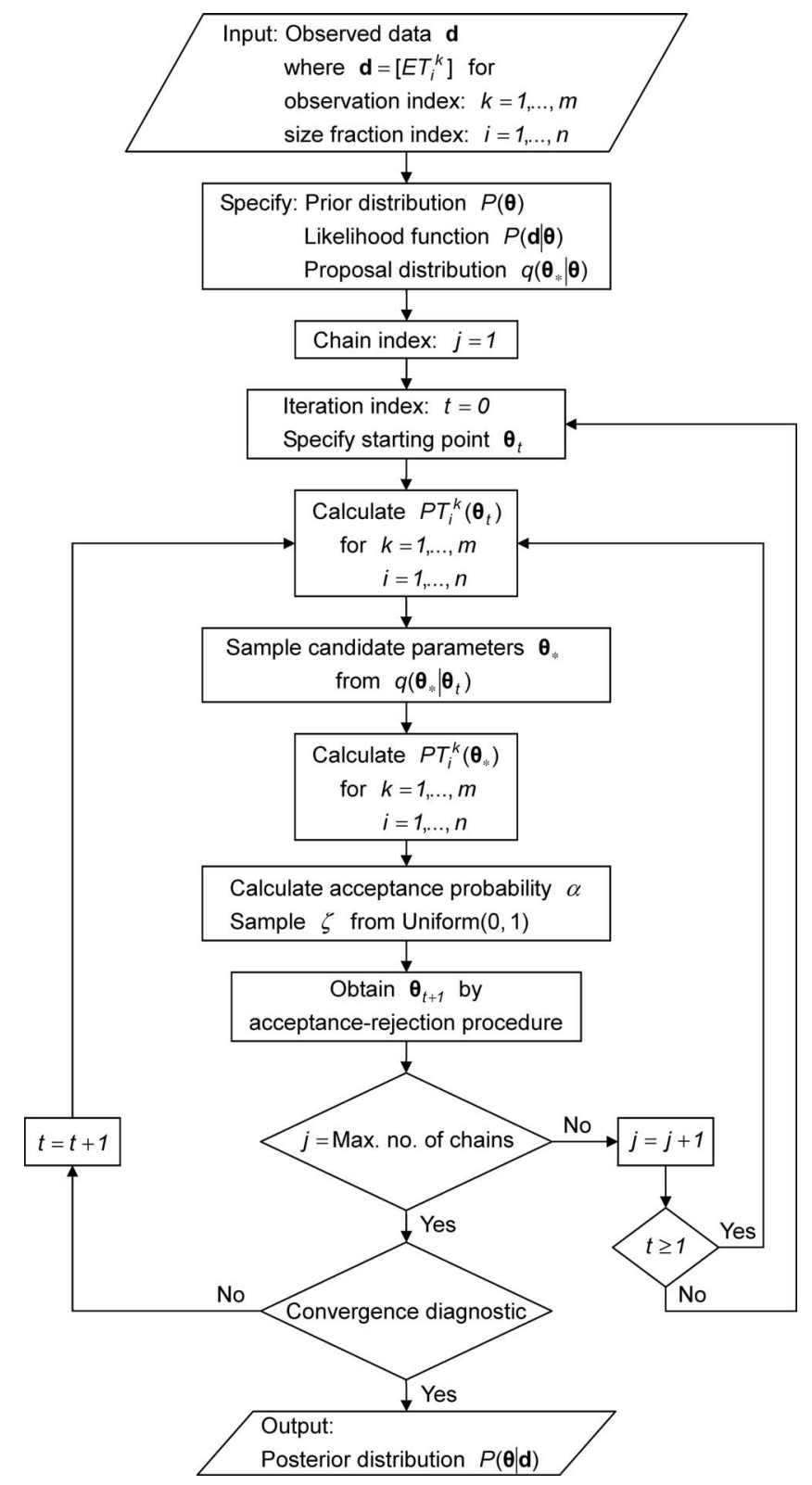

Fig. 1. Flowchart of multiple-chain MCMC simulations

\section{Materials}

Two sets of data were compiled and used as the input material for parameter updating. The first data set included the entrainment probabilities observed in a gravel-bed flume with grain sizes ranging from 1.68 to $11 \mathrm{~mm}$ (Wu and Yang 2004b). Six observations (Runs C-2-C-7) were included in this data set (Table 1). The bed shear stress $\tau_{0}$ varied between 2.16 and $4.75 \mathrm{~Pa}$; values of $k_{s}^{+}$ ranged from 740 to 1,200 , all in rough regimes. The partial transport observed in these runs was reflected by the relatively low probabilities of entrainment, especially for Runs C-2 and C-4 in which the values of $\tau_{0}$ were smallest. The second set included the entrainment probabilities observed in four sediment mixtures with grain sizes ranging between 0.042 and $4.472 \mathrm{~mm}$ (Sun and Donahue 2000). Nine observations were included in this data set and shown in Table 2, where the entrainment probabilities of the size fractions between 0.22 and $2.45 \mathrm{~mm}$ are summarized. The bed shear stress $\tau_{0}$ covered a range between 0.57 and $1.6 \mathrm{~Pa}$, the 
Table 1. Compiled Data from Gravel-Bed Experiments

\begin{tabular}{|c|c|c|c|c|c|}
\hline Run & $\tau_{0}(\mathrm{~Pa})$ & $k_{s}^{+}$ & $D_{i}(\mathrm{~mm})$ & $p_{i}(\%)$ & $E T_{i}$ \\
\hline \multirow[t]{6}{*}{$\mathrm{C}-2$} & 2.16 & 740 & 1.68 & 1.3 & \\
\hline & & & 2.59 & 6.6 & \\
\hline & & & 3.67 & 11.8 & \\
\hline & & & 5.04 & 10.7 & \\
\hline & & & 7.78 & 39.3 & \\
\hline & & & 11 & 30.3 & 0.018 \\
\hline \multirow[t]{6}{*}{$\mathrm{C}-3$} & 3.76 & 1,040 & 1.68 & 0.9 & 0.176 \\
\hline & & & 2.59 & 6.1 & 0.138 \\
\hline & & & 3.67 & 5.8 & 0.096 \\
\hline & & & 5.04 & 9.6 & 0.061 \\
\hline & & & 7.78 & 40.6 & 0.058 \\
\hline & & & 11 & 37.1 & \\
\hline \multirow[t]{6}{*}{ C-4 } & 3.08 & 940 & 1.68 & 0.8 & \\
\hline & & & 2.59 & 8.7 & \\
\hline & & & 3.67 & 8.2 & \\
\hline & & & 5.04 & 8.8 & 0.021 \\
\hline & & & 7.78 & 39.5 & \\
\hline & & & 11 & 33.9 & \\
\hline \multirow[t]{6}{*}{ C-5 } & 4.46 & 1,130 & 1.68 & 0.8 & 0.229 \\
\hline & & & 2.59 & 7.7 & 0.164 \\
\hline & & & 3.67 & 7.2 & 0.113 \\
\hline & & & 5.04 & 8.6 & 0.087 \\
\hline & & & 7.78 & 39.2 & 0.039 \\
\hline & & & 11 & 36.5 & 0.033 \\
\hline \multirow[t]{6}{*}{ C-6 } & 4.75 & 1,200 & 1.68 & 0.8 & 0.306 \\
\hline & & & 2.59 & 9.4 & 0.229 \\
\hline & & & 3.67 & 5.7 & 0.171 \\
\hline & & & 5.04 & 7.6 & 0.133 \\
\hline & & & 7.78 & 36.2 & 0.057 \\
\hline & & & 11 & 40.2 & 0.043 \\
\hline \multirow[t]{6}{*}{ C-7 } & 4.06 & 1,080 & 1.68 & 1.7 & 0.177 \\
\hline & & & 2.59 & 13.4 & 0.144 \\
\hline & & & 3.67 & 11 & 0.107 \\
\hline & & & 5.04 & 9.7 & 0.082 \\
\hline & & & 7.78 & 34.3 & 0.065 \\
\hline & & & 11 & 29.8 & 0.055 \\
\hline
\end{tabular}

corresponding values of $k_{s}^{+}$ranged from 26 to 56, all in transitional regimes $\left(5<k_{s}^{+}<70\right)$. The first data set was used for updating the rough-regime parameters, whereas the second set was used for updating parameters in transitional regimes.

\section{Results and Discussion}

In the first part of this section, the results of univariate MCMC simulations are presented. The velocity coefficient $C$ was updated using different numbers of chains, scale factors $s$, prior distributions, correlation coefficients $R_{u v}$, and numbers of input data, and their effects on the outcomes of MCMC were explored. In the second part, multivariate MCMC were performed to simultaneously update the values of $C$ and six statistical moments of the GC PDF. The results of univariate and multivariate MCMC were compared. Last, the effect of applying the updated parameters on the prediction of entrainment probabilities was demonstrated.

\section{Sensitivity Analyses}

Sensitivity analyses were performed in this section to explore the effects of chain number, scale factor $s$, prior distribution, correlation coefficient $R_{u v}$, and additional input data on the outcomes of MCMC. Univariate MCMC simulations were carried out to update the velocity coefficient $C$, whereas the six statistical moments of the GC PDF remained constant as given in Eq. (9a). The gravel-bed data (C-2-C-7) were used as the pool of input material.

\section{Number of Chains}

Two- and three-chain MCMC were performed to examine whether using different numbers of chains would result in different outcomes. The starting values used in the two-chain MCMC were 1 and 15 [Fig. 2(b)], whereas an extra starting value of 25 was used in the three-chain MCMC [Fig. 2(a)]. Here, for simplicity, a single set of data (C-5) was used as the input. Chains were run for sufficiently long to confirm that they had indeed converged. The evolution of $R$ (PSRF) to unity is shown in Fig. 3, where it is demonstrated that two-chain MCMC reached equilibrium after approximately 1,000 iterations, whereas the three chains required more than 1,500 iterations to be fully mixed. The faster convergence of two-chain MCMC was also observed in Fig. 2(b), as compared to the three chains shown in Fig. 2(a). To further examine the efficient mixing of sampler output, the lagautocorrelations for each line of the three chains (Fig. 4) demonstrate that the autocorrelations within each chain were practically negligible and confirm that convergence was efficient.

The posterior histograms for each line of the three chains are shown in Figs. 5(a-c), and the histogram derived from all three lines is shown in Fig. 5(d), where the corresponding best-fit PDF are Normal(14.6, 4.8), BetaGeneral(6.0, 2.2, 2.4, 20.6), BetaGeneral(5.6, 3.7, 7.5, 22.2), and BetaGeneral(6.6, 3.6, 4.3, 21.9), respectively. These posterior PDF are all plotted in Fig. 5(e) for an overall comparison. Although the posterior PDF of chain 1 was less similar to the other two, the three-chain PDF eliminated individual differences and may well represent the distribution pattern of the entire sampler output. The posterior PDFs of two- and three-chain MCMC are shown in Fig. 5(f), where the best-fit PDF resulting from two-chain MCMC, i.e., BetaGeneral(4.2, 3.3, 8.9, 23.4), slightly deviated from the three-chain PDF in the location of mode (17.3 versus 16.2), but had an identical probability density $(=0.15)$ at the mode. These results suggest that the outcomes of two- and three-chain MCMC were, practically speaking, not significantly different. Thus, throughout this study we use two chains to perform MCMC.

\section{Scale Factor of Proposal Distribution}

Three values of $s$ (i.e., $0.25,0.5$, and 1.0) were used to explore the influence of scale factor on the outcomes of MCMC. Two-chain simulations were carried out using an uninformed prior. The sampler outputs are shown in Figs. 2(b-d), with the corresponding evolutions of PSRF demonstrated in Fig. 3. The sampler output associated with $s=1.0$ reached equilibrium after approximately 1,500 iterations, whereas the sampler output associated with $s=0.25$ exhibited very slow mixing and the convergence criterion was almost reached after 3,000 iterations. Both of these sampler outputs, however, mixed more slowly than the output associated with $s=0.5$, which confirmed that a scale factor too cautious or too bold would result in slow convergence.

The posterior PDF of the sampler outputs associated with $s=0.5$ and 1.0 are demonstrated in Fig. 5(f), the functional form 
Table 2. Compiled Data from Experiments with Four Types of Sediment Mixture

\begin{tabular}{|c|c|c|c|c|c|c|c|c|}
\hline \multicolumn{2}{|c|}{ Sediment mixture } & \multicolumn{7}{|c|}{$\tau_{0}(\mathrm{~Pa}) k_{s}^{+}$} \\
\hline$D_{i}(\mathrm{~mm})$ & $p_{i}(\%)$ & & & & & & & \\
\hline \multicolumn{2}{|c|}{ Type 1} & 0.57 & 26 & 0.64 & 28 & 0.73 & & 30 \\
\hline 0.22 & 6 & \multicolumn{2}{|c|}{0.715} & \multicolumn{2}{|c|}{0.753} & \multicolumn{3}{|c|}{0.793} \\
\hline 0.27 & 6 & \multicolumn{2}{|c|}{0.676} & \multicolumn{2}{|c|}{0.719} & \multicolumn{3}{|c|}{0.764} \\
\hline 0.35 & 14 & \multicolumn{2}{|c|}{0.627} & \multicolumn{2}{|c|}{0.674} & \multicolumn{3}{|c|}{0.726} \\
\hline 0.45 & 12 & \multicolumn{2}{|c|}{0.566} & \multicolumn{2}{|c|}{0.619} & \multicolumn{3}{|c|}{0.678} \\
\hline 0.55 & 12 & \multicolumn{2}{|c|}{0.513} & \multicolumn{2}{|c|}{0.57} & \multicolumn{3}{|c|}{0.635} \\
\hline 0.69 & 12 & \multicolumn{2}{|c|}{0.449} & \multicolumn{2}{|c|}{0.51} & \multicolumn{3}{|c|}{0.58} \\
\hline 0.89 & 8 & \multicolumn{2}{|c|}{0.377} & & & & 0.515 & \\
\hline 1.22 & 8 & & & & & & 0.429 & \\
\hline 1.73 & 5 & & & & & & 0.33 & \\
\hline 2.45 & 5 & & & & & & 0.235 & \\
\hline & & 1.11 & 36 & 0.86 & 32 & 1.1 & & 36 \\
\hline 0.22 & 6 & & & & & & 0.877 & \\
\hline 0.27 & 6 & & & & & & 0.86 & \\
\hline 0.35 & 14 & & & & & & 0.837 & \\
\hline 0.45 & 12 & & & & & & 0.807 & \\
\hline 0.55 & 10 & & & & & & 0.780 & \\
\hline 0.69 & 11 & & & & & & 0.744 & \\
\hline 0.89 & 8 & & & & & & 0.699 & \\
\hline 1.22 & 9 & & & & & & 0.634 & \\
\hline 1.73 & 4 & & & & & & 0.552 & \\
\hline 2.45 & 5 & & & & & & 0.459 & \\
\hline & & 1.55 & 41 & 1.26 & 37 & & & \\
\hline 0.22 & 6 & & & & & & & \\
\hline 0.27 & 6 & & & & & & & \\
\hline 0.35 & 14 & & & & & & & \\
\hline 0.45 & 12 & & & & & & & \\
\hline 0.55 & 4 & & & & & & & \\
\hline 0.69 & 14 & & & & & & & \\
\hline 0.89 & 2 & & & & & & & \\
\hline 1.22 & 9 & & & & & & & \\
\hline 1.73 & 7 & & & & & & & \\
\hline 2.45 & 4 & & & & & & & \\
\hline & & 1.6 & 56 & & & & & \\
\hline 0.22 & 3 & & & & & & & \\
\hline 0.27 & 8 & & & & & & & \\
\hline 0.35 & 7 & & & & & & & \\
\hline 0.45 & 8 & & & & & & & \\
\hline 0.55 & 5 & & & & & & & \\
\hline 0.69 & 9 & & & & & & & \\
\hline 0.89 & 4 & & & & & & & \\
\hline 1.22 & 9 & & & & & & & \\
\hline 1.73 & 7 & & & & & & & \\
\hline 2.45 & 11 & & & & & & & \\
\hline
\end{tabular}

of the latter is Logistic $(15.8,1.8)$. The PDF of the sampler output associated with $s=0.25$ is not shown because it had not fully converged. Practically speaking, the difference between the posterior PDF associated with $s=0.5$ and 1.0 was not significant, with their modes located at 17.3 and 15.8, and the corresponding probability densities being 0.15 and 0.14 , respectively. The results suggest that the scale factor of proposal affects significantly the rate of convergence, but not so pronouncedly the posterior distributions.

\section{Prior Distribution}

Two alternative priors, i.e., a semiinformed normal prior $N(8.05,3.1)$ with its mean and variance derived from the reported lower and upper bounds (Nikora et al. 2001) and a normal posterior $N(17.3,5.5)$ resulting from uninformed prior, were used for exploring the effect of prior on the outcomes of MCMC. The sampler outputs resulting from these alternative priors (denoted as normal prior-1 and prior-2, respectively) are shown in Figs. 2(e and f), which are to be compared with the output resulting from 

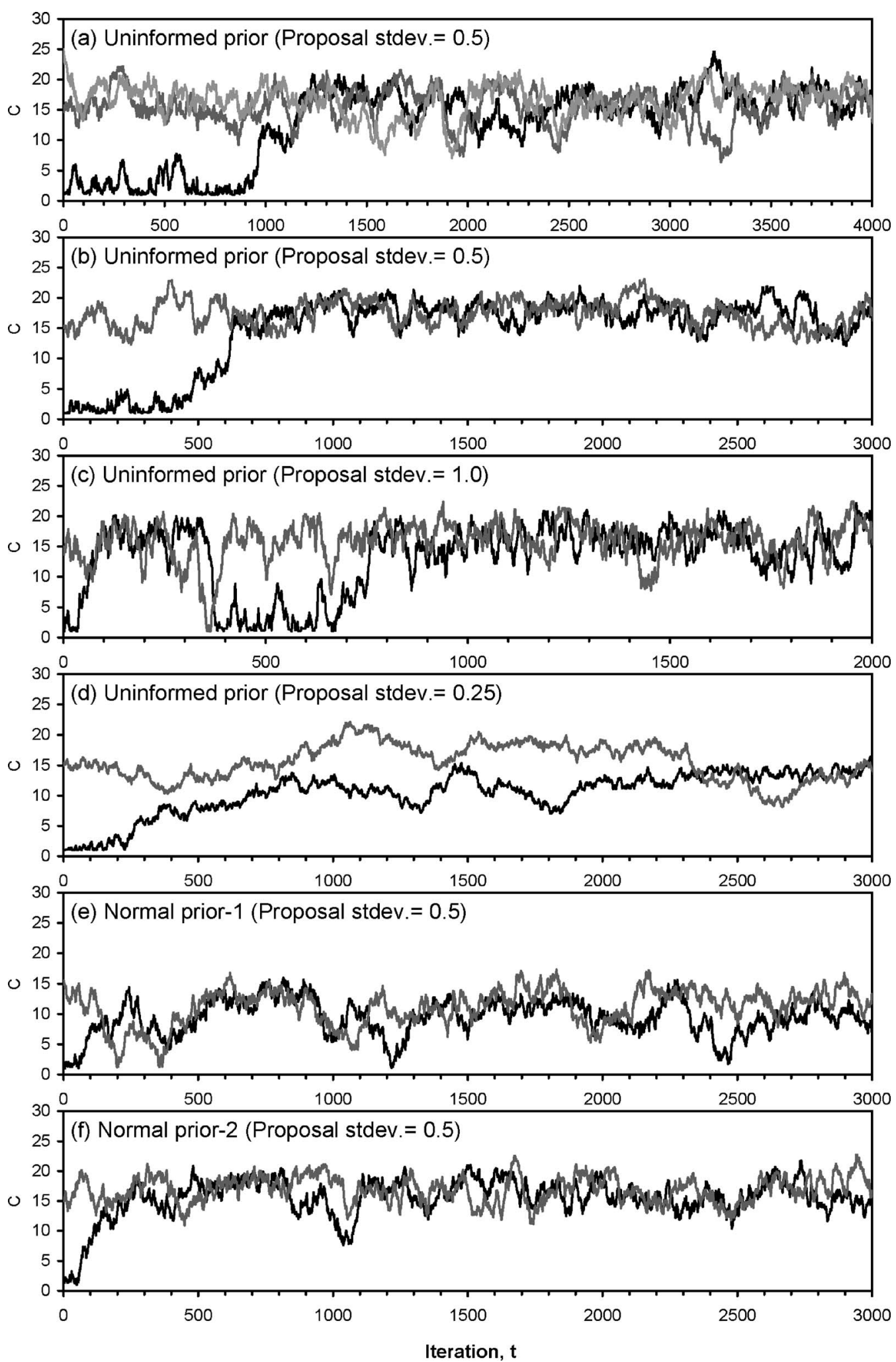

Fig. 2. Sampler outputs of velocity coefficient $C$ resulting from univariate MCMC using (a) three chains, uninformed prior, and proposal scale factor $=0.5$; (b) two chains, uninformed prior, and proposal scale factor $=0.5$; (c) two chains, uninformed prior, and proposal scale factor $=1.0$; (d) two chains, uninformed prior, and proposal scale factor $=0.25$; (e) two chains, normal prior $N(8.05,3.1)$, and proposal scale factor $=0.5$; and (f) two chains, normal prior $N(17.3,5.5)$, and proposal scale factor $=0.5$ 


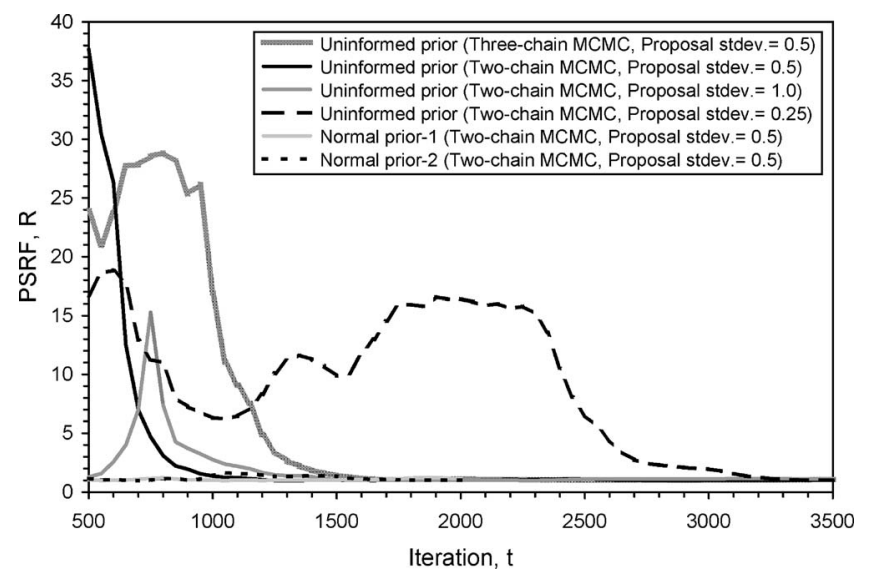

Fig. 3. Evolutions of potential scale reduction factor (PSRF) $R$ for sampler outputs shown in Fig. 2

uninformed prior [Fig. 2(b)]. The results revealed that convergence of sampler outputs was achieved much faster as normal priors were used. For both of the simulations using normal priors, equilibrium was reached prior to 500 iterations (Fig. 3). Although normal prior- 1 and prior- 2 were different in values of mean and variance, their output results consistently demonstrated better convergence than the output resulting from uninformed prior.

The posterior distributions resulting from normal prior-1 and prior-2 are demonstrated in Fig. 5(f) along with other posteriors discussed earlier. The posterior PDF resulting from normal prior-1 and prior-2, i.e., BetaGeneral(11.5, 3.5, -10.0, 16.9) and BetaGeneral(4.3, 5.2, 10.0, 24.0), were significantly different, with their modes located at 11.8 and 16.1, and the corresponding probability densities being 0.14 and 0.17 , respectively. It is speculated that the smaller posterior mode resulting from normal prior-1 was mainly attributed to its smaller prior mode $(=8.05)$, whereas the greater posterior mode resulting from normal prior-2 was attributable to its greater prior mode $(=17.3)$. Further, the highest probability density corresponding to the posterior mode was observed for the outcome resulting from an informed prior (i.e., normal prior-2). As this informed prior was a posterior of uninformed prior, the posterior of normal prior- 2 was also similar to those resulting from uninformed priors. However, the highest probability density corresponding to the posterior mode may imply that uncertainty reduction was enhanced by incorporating more prior information.

The results suggest that convergence of sampler outputs is achieved faster if informed priors are used. The posterior distri- butions are significantly affected by input priors, and parameter uncertainty is reduced as informed priors are incorporated into the MCMC.

\section{Correlation Coefficient of the GC Joint PDF}

Three typical values of $R_{u v}$ (i.e., $-0.4,-0.45$, and -0.5 ) were used to examine whether the outcomes of MCMC were influenced by the correlation coefficient of the GC joint PDF. The scale factor $s=0.5$ and uninformed prior were used in these simulations. The sampler outputs resulting from these values of $R_{u v}$ are shown in Figs. 6(a-c), with the corresponding evolution of PSRF given in Fig. 6(d). Convergence of the sampler output resulting from $R_{u v}=-0.4$ was the fastest (convergence criterion was met prior to 500 iterations), whereas convergence of the sampler output resulting from $R_{u v}=-0.5$ was the slowest (convergence criterion was met after 2,000 iterations). The results indicate that if streamwise and vertical fluctuations are more correlated, a greater number of iterations are required to reach a stationary posterior. Such an outcome was probably due to the correlation between streamwise and vertical fluctuations being a constraint imposed on MCMC. Given a higher $R_{u v}$, the values of $u^{\prime}$ and $v^{\prime}$ would be restricted in narrower ranges such that a more suitable value of $C$ must be drawn from the proposal to yield an acceptance probability $\alpha$ that would allow the chains to move forward.

The posterior distributions of the sampler outputs resulting from three values of $R_{u v}$ are shown in Fig. 7, where the best-fit PDF for $R_{u v}=-0.4$ and -0.5 were $\operatorname{Logistic}(16.0,1.2)$ and BetaGeneral $(8.1,4.4,4.9,22.5)$, respectively. The results given in Figs. 6 and 7 revealed that the rate of convergence does not necessarily coincide with the degree of uncertainty reduction. With an uninformed prior used in these runs, the reduction of parameter uncertainty may be evaluated with the shape of the posterior. The posterior PDF resulting from $R_{u v}=-0.4$ had a highest probability density $(=0.21)$ corresponding to its mode $(=16.0)$, whereas the posterior PDF resulting from $R_{u v}=-0.45$ had a lowest probability density $(=0.15)$ corresponding to its mode $(=17.3)$. The posterior PDF resulting from $R_{u v}=-0.5$ had a probability density of 0.17 corresponding to its mode at 16.8 , both the mode and probability density were between those values associated with $R_{u v}=-0.4$ and -0.45 . Because $R_{u v}$ and $C$ are both physical parameters of the sediment entrainment model rather than stochastic parameters of MCMC, we speculate that the outcomes shown in Fig. 7 were related to the physical setting of the experiment, i.e., for Run C-5 the near-bed values of $R_{u v}$ were probably most dominated by those close to -0.4 and least dominated by those close to -0.45 .
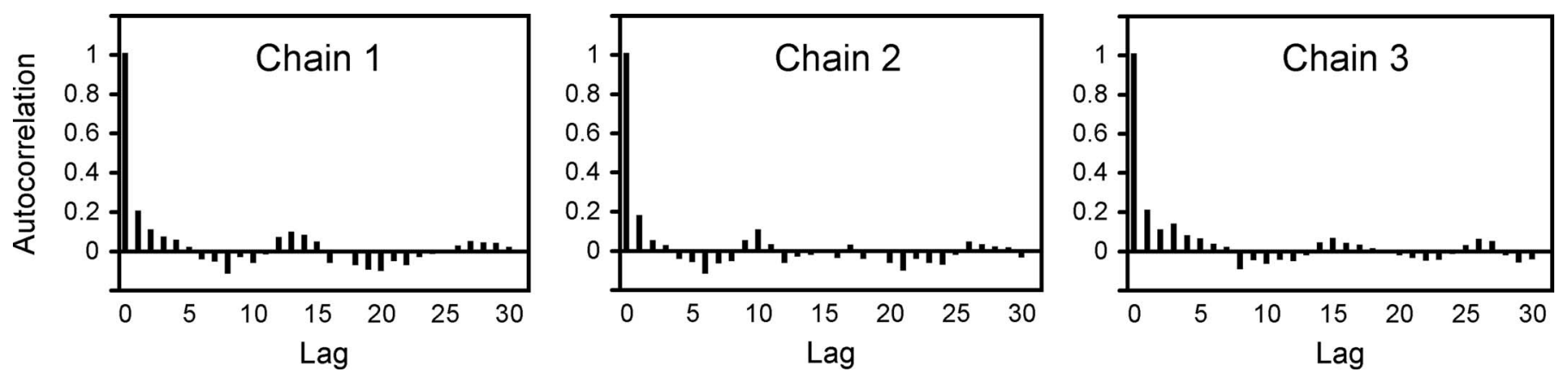

Fig. 4. Lag-autocorrelation plots for the three chains shown in Fig. 2(a) 

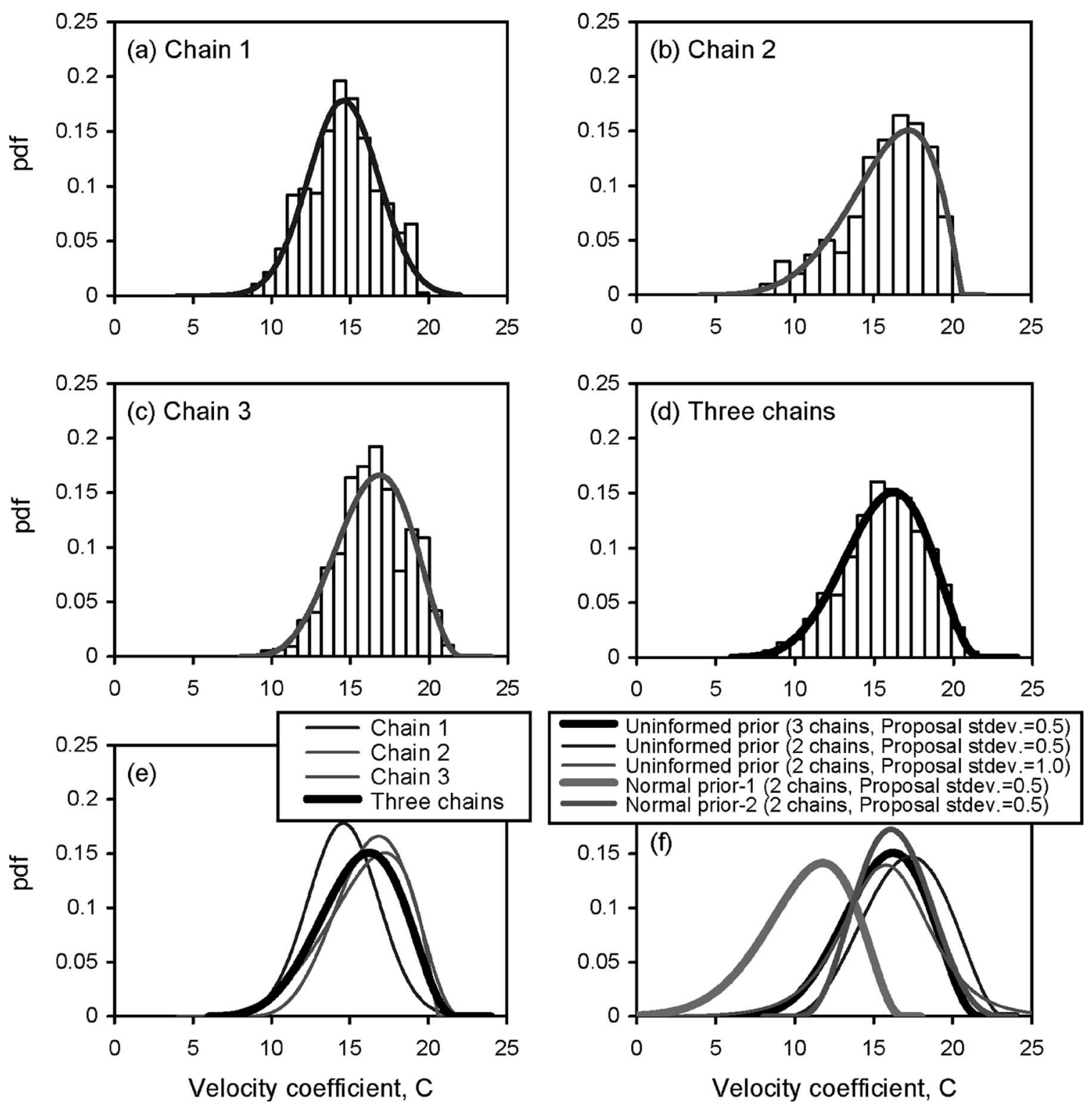

Fig. 5. Posterior histograms and best-fit PDFs of the three chains shown in Fig. 2(a) are demonstrated individually and collectively in (a)-(e) for comparison. Best-fit PDFs of the sampler outputs shown in Fig. 2 are demonstrated in (f) for comparison.

As $R_{u v}$ varies as a function of local turbulence and bed characteristics that is not fully understood at this moment $(\mathrm{Wu}$ and Jiang 2007), throughout this study a constant value of $R_{u v}=-0.45$ was used, leaving a largest uncertainty in $C$ to be resolved via multiple-input and multivariate Bayesian updating.

\section{Number of Input Data}

Bayesian updating depends crucially on additional data available, thus the number of input data should have an important influence on the results of MCMC. To explore this, we used one set of data (C-5, C-6, or C-7), two sets of data (C-5+C-6, C-5+C-7, or C-6+C-7), three sets of data $(\mathrm{C}-5-\mathrm{C}-7)$, and six sets of data (C$2-\mathrm{C}-7)$ as the inputs to MCMC. The scale factor $s=0.5$ and uninformed prior were used in these simulations. The posterior PDF resulting from different numbers of input data sets are shown in Fig. 8 and Table 3, with the posterior modes and standard deviations also listed in Table 3. Generally, the posterior PDF were distributed in much narrower ranges if more than two sets of input data were used. For the posterior PDF resulting from one set of input data, the modes ranged between 17.3 and 19.5, with the standard deviations consistently greater than 2.7 . For the posterior PDF resulting from two data sets, the modes were concentrated in the range between 17.2 and 18.2, with the standard deviations reduced to the values less than 2.0. As the number of data sets increased from 3 to 6 , the posterior mode varied slightly from 17.9 to 18.1 , with the standard deviation further reduced to 1.4. These results indicate that the parameter uncertainty reduces with increasing number of input data. So far, MCMC simulations were carried out to update a single parameter $C$. The values of $C$ derived from univariate $\mathrm{MCMC}$ were, however, consistently greater than those reported by previous investigators (e.g., Ligrani and Moffat 1986; Bandyopadhyay 1987; Bridge and Bennett 1992; Nikora et al. 2001; Wu and Yang 2004a), which may indicate that larger $C$ values would be obtained when the other parameters remain fixed, and also raise the need to perform multivariate Bayesian updating. 

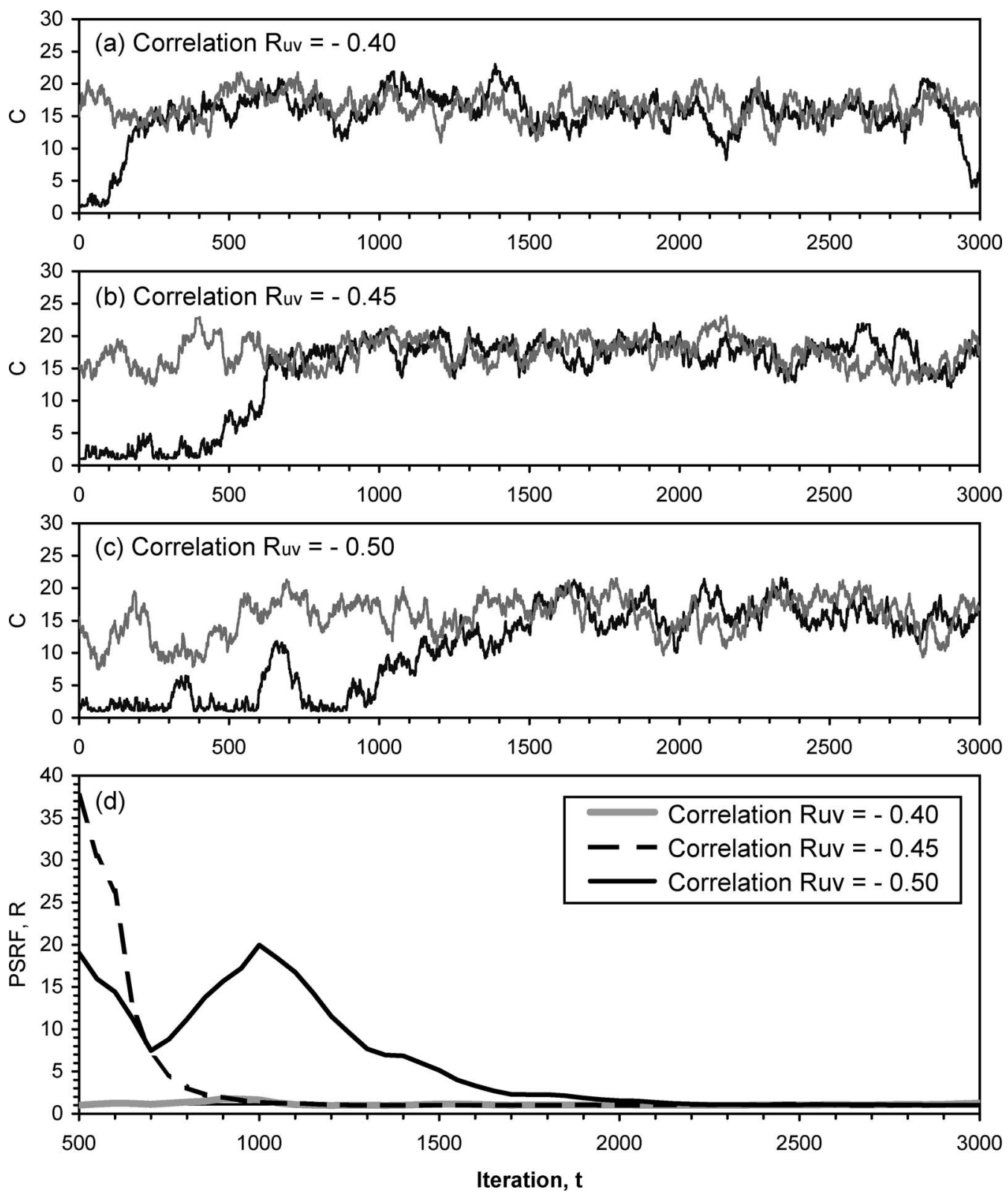

Fig. 6. Sampler outputs of velocity coefficient $C$ resulting from correlation coefficient $R_{u v}=-0.4,-0.45$, and -0.5 are shown in (a) - (c); evolutions of potential scale reduction factor (PSRF) $R$ are shown in (d)

\section{Multivariate Bayesian Updating}

Multivariate MCMC simulations were carried out in this study to simultaneously update the velocity coefficient $C$ and statistical moments of the GC joint pdf. To accelerate the rate of convergence, here a normal posterior $N(17.3,5.5)$, derived using uninformed prior and single input data set (C-5), was used as the prior of $C$ (note that this normal PDF was used earlier as an alternative prior), and a normal joint PDF was used as the prior of the statistical moments, with the mean vector and variance matrix specified in Eq. (9). The starting values were taken to be (prior mean $) \pm\left(5 \times\right.$ prior standard deviation). For $\sigma_{u} / u_{*}$ and $\sigma_{v} / u_{*}$, however, the lower chain was started with (prior mean $)+(3 \times$ prior standard deviation $)$, such that negative values could be avoided. The likelihood and proposals were those specified in Eqs. (10) and (11), with $s=0.5$ used in Eq. (11a). The full set of gravel-bed data (i.e., C-2-C-7) were used to update the

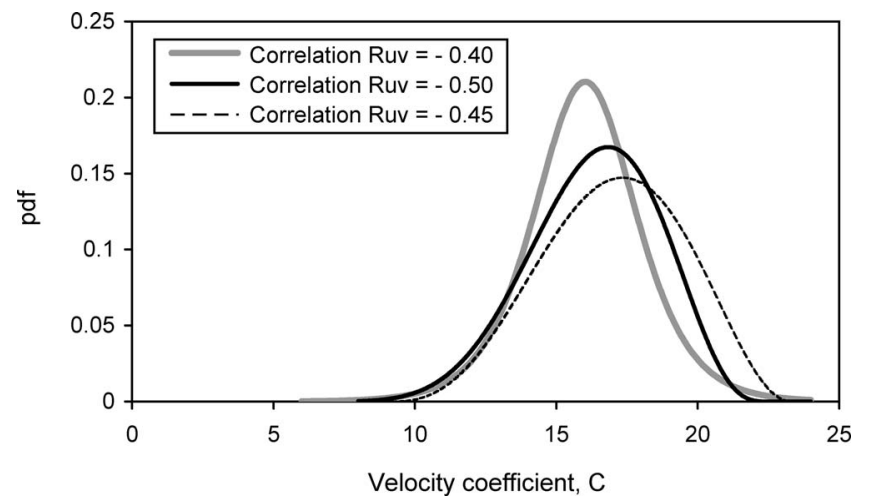

Fig. 7. Best-fit posterior PDFs of the sampler outputs shown in Fig. 6 


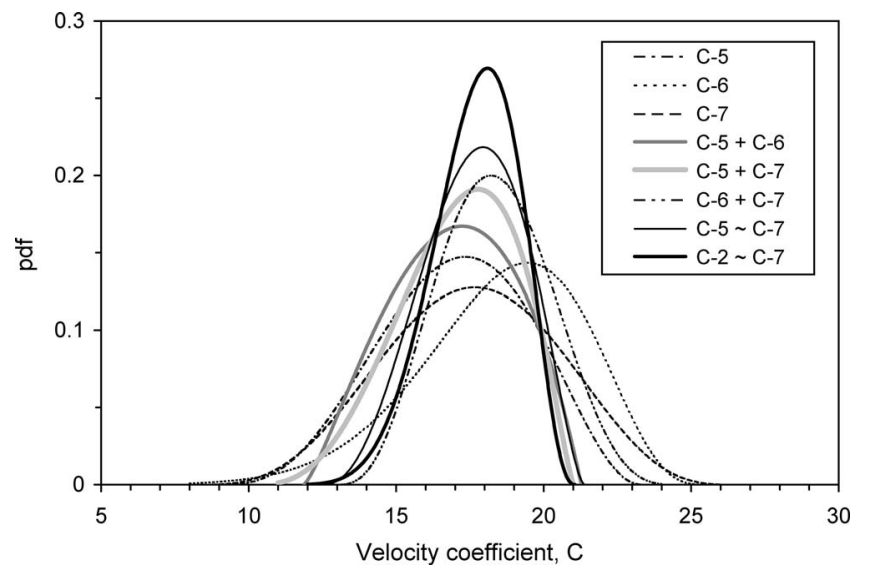

Fig. 8. Best-fit posterior PDFs of velocity coefficient $C$ resulting from different input data sets

parameters in rough regimes, whereas the compiled data given in Table 2 were used to update the parameters in transitional regimes.

For fully rough flows, the sampler outputs of all seven parameters are shown in Fig. 9, with the marginal priors and posteriors demonstrated in Fig. 10. The functional forms of posterior PDF are given in Table 4; the prior and posterior standard deviations are also given for a comparison. The distribution of $C$ was significantly modified via multivariate $\mathrm{MCMC}$, with the mode shifted from 17.3 to 10.8 and standard deviation reduced from 2.345 to 0.815 , implying some $65 \%$ reduction in uncertainty. Modifications in the distributions of six statistical moments were, however, more limited, with the reductions in standard deviation ranging from 2 to $13 \%$. Two exceptions were observed for $S_{u}$ and $S_{v}$ whose posterior standard deviations were greater than their prior values, implying that underestimation of prior uncertainties was likely to occur if sparse data were available.

For transitional regimes, $C$ varies as a function of $k_{s}^{+}$, thus the compiled data sets were used each at a time as the input to MCMC for updating the parameters associated with each $k_{s}^{+}$. The posterior modes and standard deviations resulting from multivariate MCMC are shown in Table 5, where the posterior standard deviations of $C$ resulting from univariate MCMC are given for a comparison. The posterior modes and $90 \%$ confidence intervals of $C$ resulting from univariate and multivariate MCMC are also demonstrated in Fig. 11 along with the results of several previous studies, including those of Ligrani and Moffat (1986), Bandyopadhyay (1987), Bridge and Bennett (1992), and Wu and Yang (2004a). Among these, the first three were derived for transitional
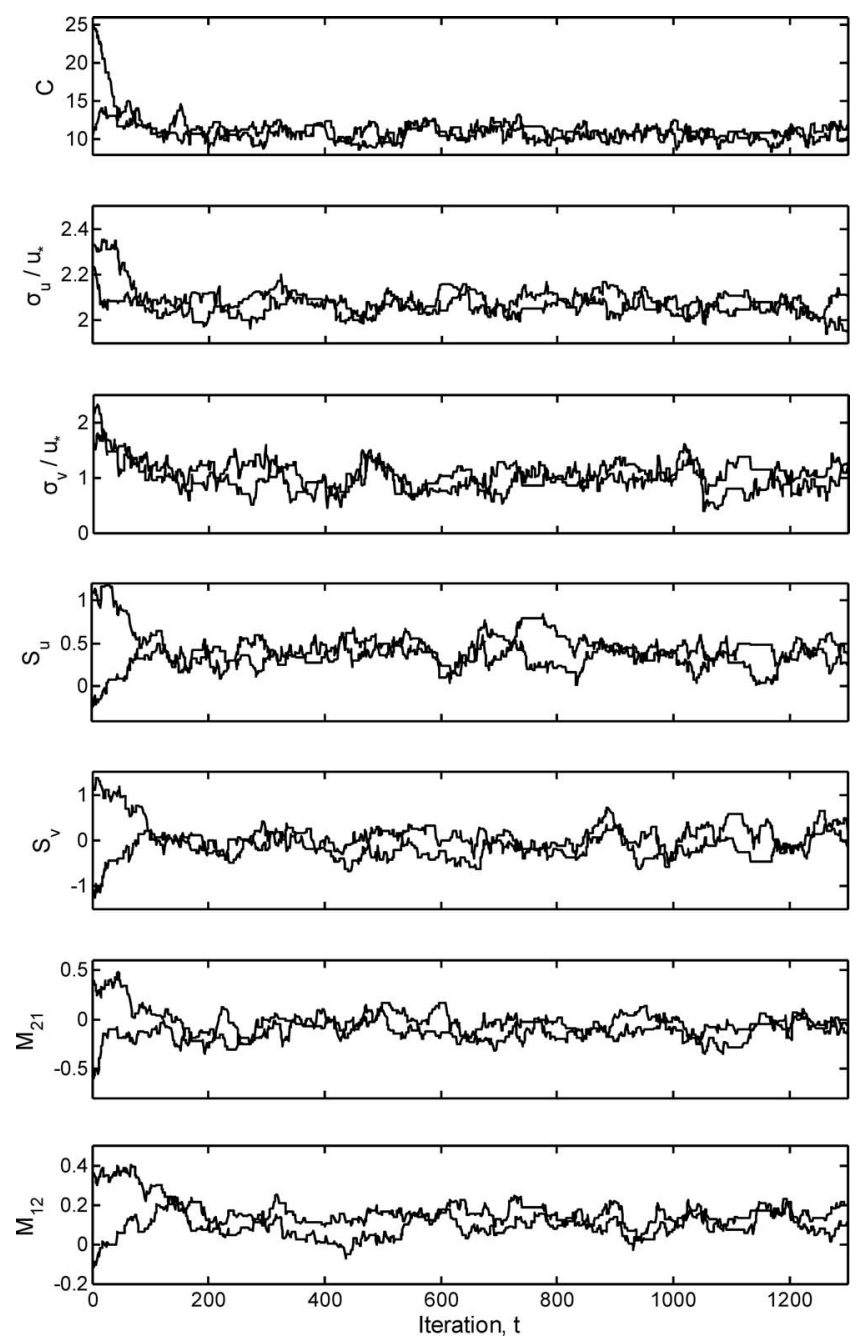

Fig. 9. Sampler outputs of velocity coefficient $C$ and six statistical moments of the GC PDF resulting from multivariate MCMC using rough-regime data

flows, and it was suggested that $C$ remains constant in fully rough flows; the last one was empirically derived from a compiled data set with $k_{s}^{+}<1,000$. As shown, the first three exhibit an increasing trend of $C$ followed by a decrease within transitional regimes, and then remain constant in fully rough regimes, whereas the last one decreases monotonically with $k_{s}^{+}$.

Fig. 11 demonstrates that the rough-regime values of $C$ resulting from multivariate MCMC were more consistent with three

Table 3. Posterior Distributions, Modes, and Standard Deviations of Velocity Coefficient Resulting from Univariate MCMC with Different Numbers of Input Data Sets

\begin{tabular}{|c|c|c|c|c|}
\hline $\begin{array}{l}\text { Number of } \\
\text { input data sets }\end{array}$ & $\begin{array}{l}\text { Input data } \\
\text { sets }\end{array}$ & Posterior PDF & Mode & $\begin{array}{l}\text { Standard } \\
\text { deviation }\end{array}$ \\
\hline \multirow[t]{3}{*}{1} & $\mathrm{C}-5$ & $\operatorname{BetaGeneral}(4.2,3.3,8.9,23.4)$ & 17.3 & 2.7 \\
\hline & C-6 & BetaGeneral $(18.7,4.9,-8.8,25.7)$ & 19.5 & 2.8 \\
\hline & $\mathrm{C}-7$ & BetaGeneral $(4.8,4.5,8.0,26.6)$ & 17.7 & 2.9 \\
\hline \multirow[t]{3}{*}{2} & $\mathrm{C}-5+\mathrm{C}-6$ & $\operatorname{BetaGeneral}(2.3,2.0,11.9,21.3)$ & 17.2 & 2.0 \\
\hline & $\mathrm{C}-5+\mathrm{C}-7$ & BetaGeneral $(4.0,2.3,10.1,21.0)$ & 17.8 & 1.9 \\
\hline & $\mathrm{C}-6+\mathrm{C}-7$ & BetaGeneral $(4.2,4.9,12.9,24.7)$ & 18.2 & 1.9 \\
\hline 3 & $\mathrm{C}-5-\mathrm{C}-7$ & $\operatorname{BetaGeneral}(3.6,2.6,12.4,21.4)$ & 17.9 & 1.7 \\
\hline 6 & $\mathrm{C}-2-\mathrm{C}-7$ & BetaGeneral $(7.9,3.6,10.2,21.1)$ & 18.1 & 1.4 \\
\hline
\end{tabular}



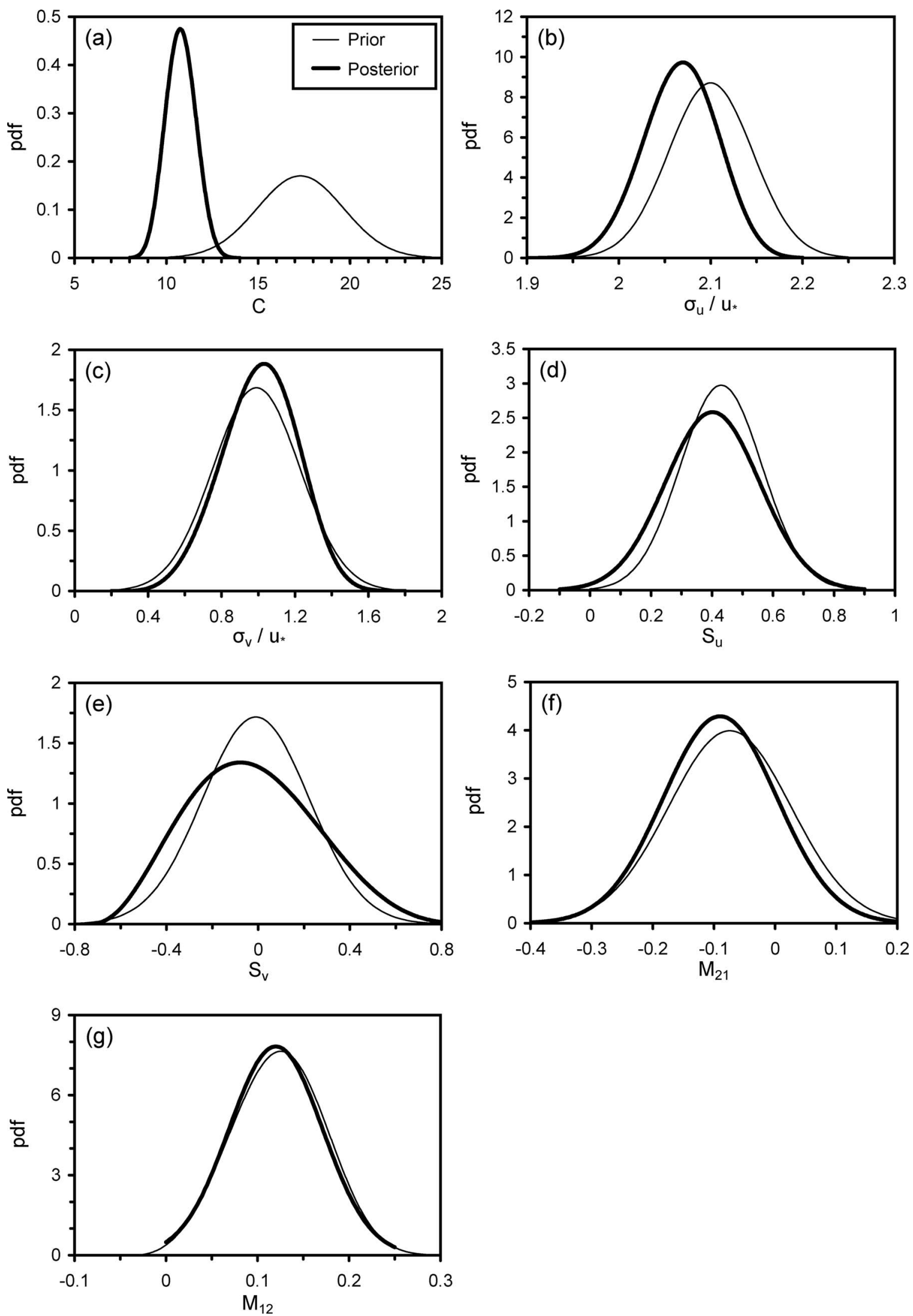

Fig. 10. Prior and posterior PDFs of the sampler outputs shown in Fig. 9 
Table 4. Posterior Distributions of Parameters Resulting from Multivariate MCMC and Comparison of Prior and Posterior Standard Deviations (for Fully Rough Flows)

\begin{tabular}{|c|c|c|c|c|}
\hline \multirow[b]{2}{*}{ Parameter } & \multirow[b]{2}{*}{ Posterior PDF } & \multicolumn{3}{|c|}{ Standard deviation } \\
\hline & & Prior & Posterior & $\left(\right.$ Change $\left.^{\mathrm{a}}\right)$ \\
\hline$C$ & BetaGeneral $(10.9,12.8,7.1,15.2)$ & 2.345 & 0.815 & $(-65 \%)$ \\
\hline$\sigma_{u} / u_{*}$ & BetaGeneral(19.3, 13.8, 1.8, 2.3) & 0.046 & 0.040 & $(-13 \%)$ \\
\hline$\sigma_{v} / u_{*}$ & Weibull(4.2, 0.84) & 0.237 & 0.206 & $(-13 \%)$ \\
\hline$S_{u}$ & $\operatorname{Normal}(0.40,0.15)$ & 0.134 & 0.154 & $(+15 \%)$ \\
\hline$S_{v}$ & $\operatorname{BetaGeneral}(3.6,5.4,-0.74,1.1)$ & 0.232 & 0.278 & $(+20 \%)$ \\
\hline$M_{21}$ & $\operatorname{Normal}(-0.090,0.093)$ & 0.100 & 0.093 & $(-7 \%)$ \\
\hline$M_{12}$ & Weibull(3.6, 0.18) & 0.051 & 0.050 & $(-2 \%)$ \\
\hline
\end{tabular}

${ }^{\mathrm{a}}$ Percentage change between prior and posterior standard deviations.

previous results, whereas those resulting from univariate MCMC were much greater. The $90 \%$ confidence interval resulting from multivariate MCMC was $43 \%$ smaller than that resulting from univariate MCMC. For transitional regimes, the discrepancies between the outcomes of univariate and multivariate MCMC were not as significant as those for rough regimes, with the reductions in standard deviations ranging from 8 to $35 \%$ (Table 5), implying that an average of $22 \%$ reduction in uncertainty was achieved by multivariate MCMC. It is also revealed in Fig. 11 that the transitional-regime posterior modes exhibited a variation trend similar to those of three previous results, i.e., an initial increasing trend with $k_{s}^{+}$followed by a decreasing one. However, the variation trend of the posterior modes was much steeper. For $32<k_{s}^{+}$ $<70$, the posterior values of $C$ were consistently greater than previous results; for $28<k_{s}^{+}<32$, the posterior values appeared to coincide with three previous results; whereas for $k_{s}^{+}<28$, the posterior values were much closer to the result of $\mathrm{Wu}$ and Yang (2004a).

For transitional regimes, the posterior modes and standard deviations of the four statistical moments (Table 5) revealed that the differences between prior and posterior modes were almost neg- ligible. The changes between prior and posterior standard deviations were also limited, with the average changes ranging between -4 and $-11 \%$. Compared to the above-mentioned $22 \%$ reduction in the uncertainty of $C$, the modifications in the transitional-regime statistical moments were rather modest, similar to the situations observed for rough regimes. However, increases in the posterior standard deviations were observed sporadically, which again could be attributed to the sparse data available for estimating the prior uncertainties.

\section{Application to Prediction of Entrainment Probabilities}

In this section, the updated parameters were applied in the sediment entrainment model to demonstrate their practical effect on the prediction of entrainment probabilities. To this end, the modes of the prior and posterior distributions were used separately as the parameter values for numerical simulations. For the coefficient $C$, the mode of the normal prior $N(8.05,3.1)$ was used as the prior parameter, whereas the posterior modes resulting from multivariate MCMC (see Fig. 11) were used as the posterior parameters for different values of $k_{s}^{+}$. For the statistical moments of the GC joint

Table 5. Posterior Modes and Standard Deviations of Parameters Resulting from Multivariate MCMC (for Transitional Flows)

\begin{tabular}{|c|c|c|c|c|c|c|c|c|c|c|}
\hline \multirow[b]{2}{*}{ Parameter } & & \multirow[b]{2}{*}{ Prior } & \multicolumn{8}{|c|}{ Posterior for $k_{s}^{+}=$} \\
\hline & & & 26 & 28 & 30 & 32 & 36 & 37 & 41 & 56 \\
\hline \multirow[t]{3}{*}{ C } & Std. Dev ${ }^{\mathrm{a}}$ & 0.773 & 1.007 & 0.839 & 1.071 & 1.203 & 2.053 & 3.048 & 1.224 & \\
\hline & Std. Dev ${ }^{b}$ & & 0.636 & 0.657 & 0.588 & 0.789 & 0.830 & 1.805 & 2.669 & 1.124 \\
\hline & $\left(\right.$ Change $\left.^{c}\right)$ & & $(-18 \%)$ & $(-35 \%)$ & $(-30 \%)$ & $(-26 \%)$ & $(-31 \%)$ & $(-12 \%)$ & $(-12 \%)$ & $(-8 \%)$ \\
\hline \multirow{3}{*}{$\sigma_{v} / u_{*}$} & Mode & 0.99 & 0.97 & 0.94 & 0.97 & 1.05 & 1.04 & 0.97 & 1.04 & 0.96 \\
\hline & Std. dev. & 0.237 & 0.189 & 0.240 & 0.168 & 0.282 & 0.280 & 0.204 & 0.237 & 0.213 \\
\hline & $\left(\right.$ Change $\left.^{\mathrm{d}}\right)$ & & $(-20 \%)$ & $(+1 \%)$ & $(-29 \%)$ & $(+19 \%)$ & $(+18 \%)$ & $(-14 \%)$ & $(0 \%)$ & $(-10 \%)$ \\
\hline \multirow[t]{3}{*}{$S_{v}$} & Mode & -0.01 & -0.01 & 0.00 & -0.01 & 0.01 & -0.01 & 0.00 & 0.00 & 0.00 \\
\hline & Std. dev. & 0.233 & 0.222 & 0.219 & 0.221 & 0.191 & 0.183 & 0.283 & 0.196 & 0.225 \\
\hline & $\left(\right.$ Change $\left.^{\mathrm{d}}\right)$ & & $(-5 \%)$ & $(-6 \%)$ & $(-5 \%)$ & $(-18 \%)$ & $(-21 \%)$ & $(+21 \%)$ & $(-16 \%)$ & $(-3 \%)$ \\
\hline \multirow[t]{3}{*}{$M_{21}$} & Mode & -0.07 & -0.07 & -0.08 & -0.06 & -0.13 & -0.08 & -0.05 & -0.07 & -0.09 \\
\hline & Std. dev. & 0.100 & 0.090 & 0.089 & 0.079 & 0.091 & 0.087 & 0.097 & 0.096 & 0.082 \\
\hline & $\left(\right.$ Change $\left.^{\mathrm{d}}\right)$ & & $(-10 \%)$ & $(-11 \%)$ & $(-21 \%)$ & $(-9 \%)$ & $(-13 \%)$ & $(-3 \%)$ & $(-4 \%)$ & $(-18 \%)$ \\
\hline \multirow[t]{3}{*}{$M_{12}$} & Mode & 0.12 & 0.13 & 0.11 & 0.11 & 0.14 & 0.11 & 0.11 & 0.13 & 0.12 \\
\hline & Std. dev. & 0.051 & 0.049 & 0.059 & 0.047 & 0.037 & 0.055 & 0.042 & 0.049 & 0.039 \\
\hline & $\left(\right.$ Change $\left.^{\mathrm{d}}\right)$ & & $(-4 \%)$ & $(+16 \%)$ & $(-8 \%)$ & $(-27 \%)$ & $(+8 \%)$ & $(-18 \%)$ & $(-4 \%)$ & $(-24 \%)$ \\
\hline
\end{tabular}

Note: Posterior standard deviations of coefficient $C$ resulting from univariate MCMC are also listed for comparison.

${ }^{\text {a }}$ Posterior standard deviation resulting from univariate MCMC.

${ }^{\mathrm{b}}$ Posterior standard deviation resulting from multivariate MCMC.

${ }^{c}$ Percentage change between univariate and multivariate posterior standard deviations.

${ }^{\mathrm{d}}$ Percentage change between prior and posterior standard deviations. 


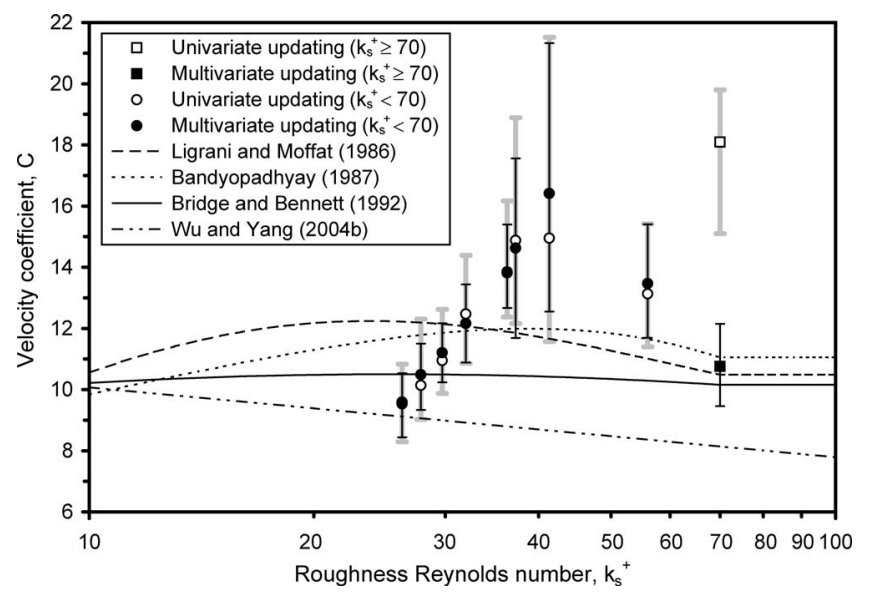

Fig. 11. Posterior modes and $90 \%$ confidence intervals of velocity coefficient $C$ resulting from univariate and multivariate MCMC. Compiled data shown in Table 2 were used for updating the constant value of $C$ in rough regimes $\left(k_{s}^{+} \geqslant 70\right)$, whereas compiled data shown in Table 3 were used for updating the variation trend of $C$ in transitional regimes $\left(k_{s}^{+}<70\right)$. Symbols and error bars denote the modes and $90 \%$ confidence intervals, respectively. Gray error bars are with empty symbols, whereas black error bars are with solid symbols. Results of four previous studies are shown for comparison.

PDF, the modes (or means) given in Eq. (9a) were used as the prior parameters, whereas the posterior modes shown in Fig. 10 and Table 5 were used as the posterior parameters for rough and transitional regimes, respectively. Comparisons of the predicted and observed entrainment probabilities are illustrated in Fig. 12, where the outcomes resulting from prior and posterior parameters are demonstrated. The entrainment probabilities predicted using the posterior parameters were more consistent with the observed data, whereas the predicted entrainment probabilities associated with prior parameters were consistently lower than the observed values. Such a result was probably due to a small value of $C$ $(=8.05)$ used as the prior parameter, given the fact that the posterior modes of $C$ deviated substantially from this prior value but the posterior modes of the statistical moments were not significantly different from their prior values. With the parameters updated for rough and transitional regimes, the sediment entrainment model was able to compute more accurately and realistically the entrainment probabilities. The global coefficient of determination $R^{2}$ increased from 0.76 to 0.91 as the prior parameters were replaced by the posterior ones, which implied a $20 \%$ improvement in the accuracy of predictions.

\section{Conclusions}

This work presents a Bayesian framework using MCMC to update the parameters of a sediment entrainment model. In the first part of this paper, univariate MCMC sensitivity analyses were performed using different numbers of chains, scale factors of proposal, prior distributions, correlation coefficients, and numbers of input data. The results reveal that the outcomes of two- and threechain MCMC were, practically speaking, not significantly different. Convergence of two-chain MCMC was, however, faster than that of three-chain MCMC. The results confirmed that a scale factor too cautious or too bold would result in slow convergence. The results also suggested that the scale factor significantly af-
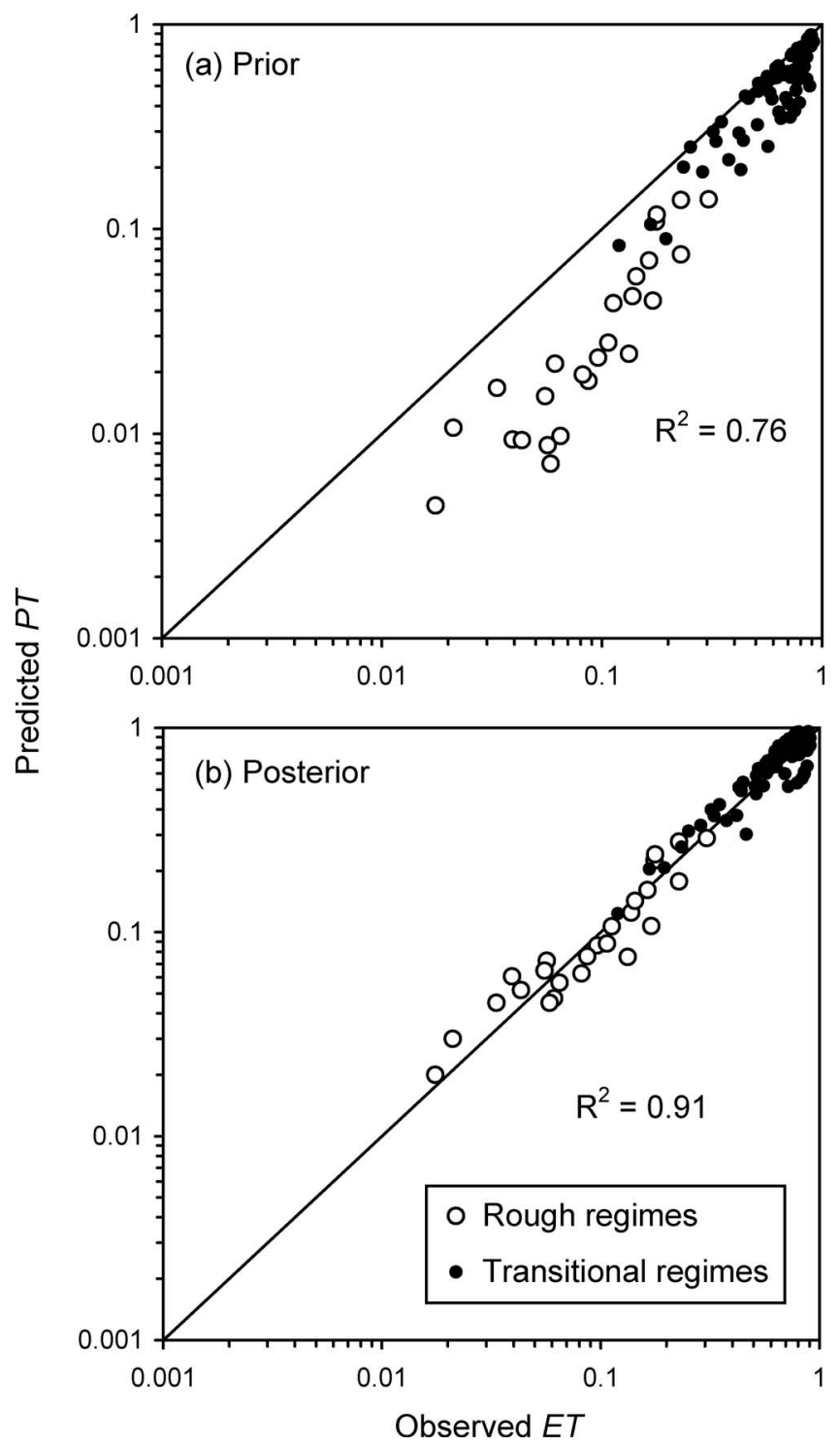

Fig. 12. Comparisons of observed data and predicted entrainment probabilities using prior and posterior parameters

fects the rate of convergence but not the posterior distributions. The sampler outputs of MCMC using informed priors converge much faster than those using an uninformed prior. The posterior distributions are significantly influenced by the patterns of priors, and the reduction of parameter uncertainty is enhanced by informed priors. The correlation coefficient of the GC PDF is a physical parameter related to the specific setting of experiments, hence is a physical constraint imposed on MCMC in which a higher correlation would require a greater number of iterations to fully mix the chains. Our results indicate that the parameter uncertainty reduces with increasing number of input data sets. The posterior PDF are generally distributed in much narrower ranges if more than two sets of input data are used.

In the second part of this study, multivariate MCMC were carried out to simultaneously update the velocity coefficient $C$ and the statistical moments of the GC PDF. For fully rough regimes, the distributions of $C$ (mean and variance) were significantly modified via multivariate MCMC. For transitional regimes, the differences between the posterior values of $C$ resulting from univariate and multivariate MCMC were not as significant as 
those for rough regimes. The posterior values of $C$ exhibited a similar but steeper variation trend compared to those of previous results. For both rough and transitional regimes, the differences between the prior and posterior distributions of the statistical moments were, however, rather limited.

In the last part of this study, the practical effect of updated parameters on the prediction of entrainment probabilities was demonstrated. The entrainment probabilities predicted using the updated parameters were more consistent with the observed values. With all the parameters updated via MCMC, the sediment entrainment model was able to compute more accurately and realistically the entrainment probabilities.

\section{Acknowledgments}

This study was supported in part by the National Science Council of Taiwan ROC. Comments from the editors and two anonymous reviewers helped to improve the clarity and completeness of this presentation.

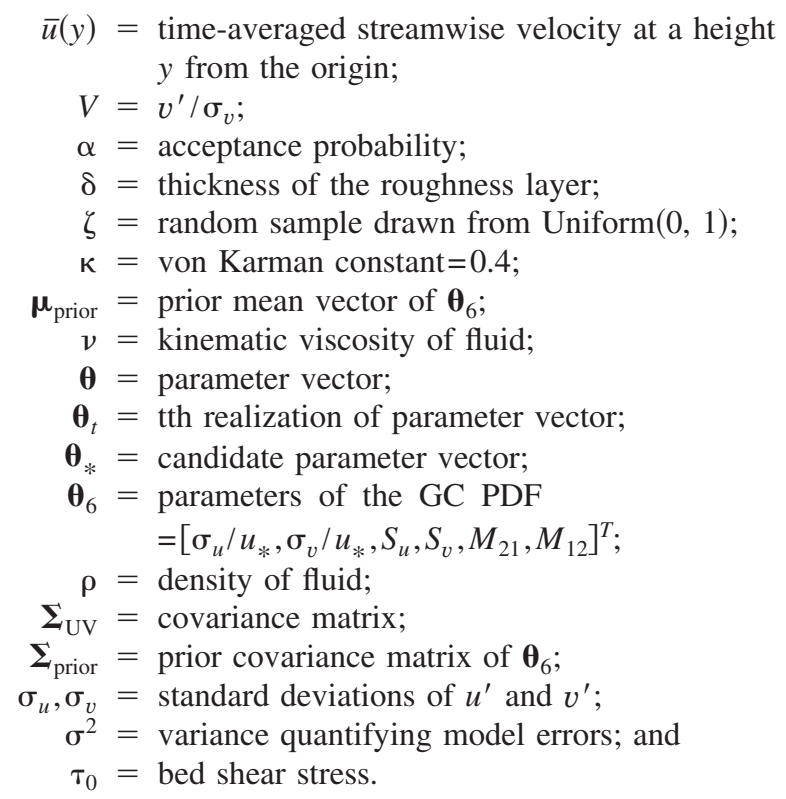

References

Balakrishnan, S., Roy, A., Ierapetritou, M. G., Flach, G. P., and Georgopoulos, P. G. (2003). "Uncertainty reduction and characterization for complex environmental fate and transport models: An empirical Bayesian framework incorporating the stochastic response surface method." Water Resour. Res., 39(12).

Bandyopadhyay, P. R. (1987). "Rough-wall turbulent boundary layers in the transitional regime." J. Fluid Mech., 180, 231-266.

Box, G. E. P. (1980). "Sampling and Bayes' inference in scientific modelling and robustness (with discussion)." J. R. Stat. Soc. Ser. A (Gen.), 143, 383-430.

Bridge, J. S., and Bennett, S. J. (1992). "A model for the entrainment and transport of sediment grains of mixed sizes, shapes, and densities." Water Resour. Res., 28(2), 337-363.

Brooks, S., and Gelman, A. (1998). "General methods for monitoring convergence of iterative simulations." J. Comput. Graph. Stat., 7, 434-455.

Draper, D. (2006). Bayesian hierarchical modeling, Springer, New York.

Gamerman, D., and Lopes, H. F. (2006). Markov chain Monte Carlo: Stochastic simulation for Bayesian inference, Chapman and Hall/ CRC, Boca Raton, Fla.

Gelfand, A. E., and Smith, A. F. M. (1990). "Sampling-based approaches to calculating marginal densities." J. Am. Stat. Assoc., 85, 399-409.

Gelman, A., Carlin, J. B., Stern, H. S., and Rubin, D. B. (2004). Bayesian data analysis, Chapman and Hall/CRC, Boca Raton, Fla.

Gilks, W. R., Richardson, S., and Spiegelhalter, D. J., eds. (1996). Markov chain Monte Carlo in practice, Chapman and Hall, London.

Ligrani, P. M., and Moffat, R. J. (1986). "Structure of transitionally rough and fully turbulent boundary layers." J. Fluid Mech., 162, 69-98.

Marshall, L., Nott, D., and Sharma, A. (2004). "A comparative study of Markov chain Monte Carlo methods for conceptual rainfall-runoff modeling." Water Resour. Res., 40.

Metropolis, N., Rosenbluth, A., Rosenbluth, M., Teller, A., and Teller, E. (1953). "Equations of state calculations by fast computing machines." J. Chem. Phys., 21, 1087-1092.

Nikora, V. I., Goring, D. G., McEwan, I., and Griffiths, G. (2001). "Spatially averaged open-channel flow over rough bed." J. Hydraul. Eng., 127(2), 123-133.

Pope, S. B. (2000). Turbulent flows, Cambridge University Press, New York.

Reis, D. S., and Stedinger, J. R. (2005). "Bayesian MCMC flood frequency analysis with historical information." J. Hydrol., 313, 97-116. 
Renard, B., Garreta, V., and Lang, M. (2006). "An application of Bayesian analysis and Markov chain Monte Carlo methods to the estimation of a regional trend in annual maxima." Water Resour. Res., 42.

Rubin, D. B. (1984). "Bayesianly justifiable and relevant frequency calculations for the applied statistician." Ann. Stat., 12, 1151-1172.

Smith, B. J. (2005). "Bayesian output analysis program (BOA) version 1.1 user's manual." Dept. of Biostatistics, Univ. of Iowa, College of Public Health, 〈http://www.public-health.uiowa.edu/boa〉.

Spall, J. C. (2003). "Estimation via Markov chain Monte Carlo." IEEE Control Syst. Mag., 34-45.

Sun, Z., and Donahue, J. (2000). "Statistically derived bedload formula for any fraction of nonuniform sediment." J. Hydraul. Eng., 126(2), $105-111$.

Wu, F.-C., and Jiang, M.-R. (2007). "Numerical investigation of the role of turbulent bursting in sediment entrainment." J. Hydraul. Eng., 133(3), 329-334.

Wu, F.-C., and Yang, K.-H. (2004a). "Entrainment probabilities of mixedsize sediment incorporating near-bed coherent flow structures." J. Hydraul. Eng., 130(12), 1187-1197.

Wu, F.-C., and Yang, K.-H. (2004b). "A stochastic partial transport model for mixed-size sediment: Application to assessment of fractional mobility." Water Resour. Res., 40(4). 